\title{
INTERVENÇÃO FISIOTERAPÊUTICA EM OSTEOARTRITE DE JOELHO E BURSITE TROCÂNTERICA: UM RELATO DE CASO
}

\section{Carolina Favero Kerche ${ }^{1}$; Alice da Silva Dorneles Bueno ${ }^{2}$; Ana Júlia Lucas \\ Bueno $^{3}$; Letícia De Freitas Irrasabal da Silva ${ }^{4}$ Aletheia Peters Bajotto ${ }^{5}$}

\section{RESUMO}

Objetivo: Descrever a abordagem fisioterapêutica da Osteoartrite de joelho e Bursite trocantérica e protocolos de tratamento bem como a avaliação pré e pósintervenção. Métodos: Relato de caso realizado na clínica de Fisioterapia da Universidade Fransciscana a partir dos atendimentos supervisionados durante sete semanas com um total de seis sessões. Foram utilizados alguns parâmetros avaliativos como a escala de Berg e o teste de alcance funcional. Resultados: Antes das sessões de fisioterapia, paciente obteve 45 pontos da escala de Berg e $11,5 \mathrm{~cm}$ no teste de alcance funcional, após seis sessões, obteve 51 pontos na escala de Berg e $31 \mathrm{~cm}$ no teste de alcance funcional, além disso, apresentou melhoras na mobilidade articular do joelho e do equilíbrio. Conclusão: A intervenção fisioterapêutica obteve melhoras no equílibrio e no alcance funcional da paciente pós as sessões de fisioterapia.

Palavras-chave: Doenças crônicas degenerativas; Envelhecimento; Fisioterapia.

Eixo Temático: Atenção Integral e Promoção à Saúde (AIPS) \section{INTRODUÇÃO}

A Osteoartrite (OA) ou osteoartrose é uma doença degenerativa da articulação, de origem não inflamatória, no qual ocorre o desgaste da cartilagem afetando os ligamentos, cápsulas, tendões e músculos, levando a alterações ósseas

\footnotetext{
${ }^{1}$ Carolina Favero Kerche - Universidade Franciscana - carolinakerche99@gmailcom

${ }^{2}$ Alice da Silva Dorneles Bueno - Universidade Franciscana - dornelesalice@hotmail.com

${ }^{3}$ Ana Júlia Lucas Bueno - Universidade Franciscana - anajuliabueno53@gmail.com

${ }^{4}$ Letícia De Freitas Irrasabal da Silva - Universidade Franciscana -leticiafreitassilva2000@gmail.com

${ }^{5}$ Aletheia Peters Bajotto - Universidade Franciscana - aletheia@bajotto.com.br
} 
como o surgimento de osteófitos. Pode causar dor, perda de função, déficit de força muscular e instabilidades articulares. Trata-se de uma patologia predominante do sexo feminino sendo uma das que mais causam incapacidades na vida dos pacientes. (RAYMUNDO et al.,2014)

Outra patologia que comumente aparecem em idosos, são as inflamações nas bursas, mais conhecidas como bursite, no qual são bolsas revestidas por líquido sinovial em seu interior, responsáveis pela diminuição do atrito entre os tendões e os músculos sobre as proeminências ósseas. (SCHWARTSMANN et al.,2014)

A bursite trocantérica é uma das mais prevalentes, sendo assim, a causa mais frequente são por micro traumas repetitivos, pelo uso ativo dos músculos inseridos no trocanter, resultando em mudanças degenerativas de tendões, músculos ou tecidos fibrosos. Além disso, tem como sintoma a dor crônica acompanhada de desconforto à palpação da região lateral do quadril, causada pela inflamação das bursas. (SCHWARTSMANN et al.,2014)

A fisioterapia em ambas patologias, tem como objetivo de reduzir o quadro álgico, melhorar a função física, diminuir encurtamentos, melhorar a mobilidade articular e reduzir o processo inflamatório no caso da bursite. (ROCHA et al.,2020). O tratamento fisioterapêutico consiste em exercícios aeróbicos para aumentar a resistência e estimular a produção de líquido sinovial, fortalecendo evitando a perda de massa muscular e consequentemente a força e alongamentos de ambos os membros com auxílio de equipamentos como bola e espaldar.

Segundo Rocha, et al. (2020), os exercícios aeróbicos associados ao fortalecimento sem carga de peso, obteve mais eficácia no alívio da dor. Portanto, exercícios que incluem equilíbrio, atividades aeróbicas e de resistência podem influenciar na melhora do quadro álgico e da função em pacientes que possuem OA.

Desta forma, este artigo apresenta um relato de caso que descreve a abordagem fisioterapêutica na OA e bursite, apresentando um protocolo de tratamento bem como a avaliação pré e pós-intervenção fisioterapêutica. 


\section{METODOLOGIA}

Trata-se de um estudo do tipo relato de caso, realizado na Clínica de Fisioterapia da Universidade Franciscana, por alunos do oitavo semestre do Curso de Fisioterapia, com supervisão de professores, durante 7 semanas, perfazendo 0 total de 6 sessões. O projeto de pesquisa foi aprovado pelo Comitê de Ética em Pesquisa da Universidade Franciscana sob o parecer de número 4.860.079.

Foram utilizados como parâmetros avaliativos, a escala de equilíbrio de berg e o teste de alcance funcional. A Escala de equilíbrio de Berg é um teste feito para avaliar a capacidade de equilíbrio estático e dinâmico, podendo prever a incidência do risco de quedas que acometem principalmente os idosos. (Jeon \& Kim,2017). É uma escala composta por 14 tarefas com pontuações de $0-4$, onde 0 é incapaz de completar a tarefa e 4 é capaz de realizar de forma independente a tarefa proposta. Além disso, o teste de alcance funcional, também tem como objetivo identificar o risco de quedas determinando a capacidade do idoso de se deslocar dentro do limite de estabilidade anterior, avaliando o controle postural através da medição com uma fita métrica. (Karuka; Silva; Am \&Navega,.2011)

\subsection{CASO CLÍNICO}

Paciente, 73 anos, sexo feminino, dona de casa, ativa, lúcida e comunicativa, residente de Santa Maria - RS. Procurou a clínica de fisioterapia através de indicação e encaminhamento médico com diagnóstico clínico desde 2019 de artrose de joelho esquerdo e bursite trocantérica no lado direito. Durante a primeira avaliação (anamnese) D.C relatou que é hipertensa fazendo uso de medicamentos como Corus 50mg e Anlodipino, diabética com o uso de Cymbi 60mg, Glifage, Insulina e forxiga, sendo sua queixa principal dor no MID em região de quadril, virilha

e joelho, além disso, referindo "quando caminho o lado direito do quadril dá umas "falhadas"'. O grau de força de acordo com a escala de Kendall foi de 3 para flexão de joelho e extensão de quadril em membro inferior direito, grau 5 em flexão de joelho e extensão de quadril em membro inferior esquerdo. Sensibilidade 
preservada, ADM passiva e ativa de MMSS conseguindo realizar igualmente nos dois lados em toda amplitude, ADM em MID diminuída devido a dor. Os instrumentos de avaliação utilizados foram escala de equilíbrio de Berg no qual a paciente obteve a pontuação de 45 e o teste de alcance funcional com pontuação de 11,5.

\subsubsection{SESSÃO FISIOTERAPIA}

\section{Primeira sessão:}

$\rightarrow$ Avaliação Inicial : Anamnese.

$\rightarrow$ Massoterapia: Liberação miofascial na região de lombar e músculo trato iliotibial em membro inferior direito.

$\rightarrow$ Eletroterapia (6'): Ultrassom no modo pulsado, frequência $1 \mathrm{MHz}$, pulso de 100 $\mathrm{Hz}$, intensidade $1 \mathrm{~W} / \mathrm{cm}^{2}$, para alívio da dor na região do quadril.

\section{Segunda sessão:}

$\rightarrow$ Aquecimento (7'): Bicicleta ergométrica

$\rightarrow$ Circuito (3 séries de 5 repetições, com intervalo de 30 segundos): Paciente sobe pela rampa e desce pela escada, indo de encontro ao step no qual irá subir e descer do mesmo.

$\rightarrow$ Exercícios de mobilidade pélvica sentado na bola suíça - (1 minuto cada exercício com intervalo de 30 segundos): Lateralização, anteversão, retroversão, circundução.

$\rightarrow$ Exercícios de propriocepção com uso da espuma proprioceptiva. (3 séries de 10 repetições cada): Plantiflexão, dorsiflexão, transferências de peso em apoio unipodal.

$\rightarrow$ Fortalecimento muscular de membros inferiores (3 séries de 12 repetições): Fortalecimento de quadríceps com uso de caneleira de $1 \mathrm{~kg}$ e fortalecimento de adutores e abdutores com caneleira de $1 \mathrm{~kg}$. 
$\rightarrow$ Eletroterapia (6'): Ultrassom no modo pulsado, frequência $1 \mathrm{MHz}$, pulso de 100 $\mathrm{Hz}$, intensidade $1 \mathrm{~W} / \mathrm{cm}^{2}$. Para alívio da dor na região do quadril e músculo trato iliotibial.

\section{Terceira sessão:}

$\rightarrow$ Aquecimento (8'): Esteira com velocidade média de 2.5)

$\rightarrow$ Exercícios de propriocepção com uso da espuma proprioceptiva. (2 séries de 15 repetições cada): Plantiflexão, dorsiflexão, transferências de peso em apoio unipodal.

$\rightarrow$ Exercícios de mobilidade pélvica sentado na bola suíça - (1 minuto cada exercício com intervalo de 30 segundos): Laterização, anteversão, retroversão e circundução.

$\rightarrow$ Fortalecimento muscular: Agachamento com auxílio da bola suíça apoiada na região de coluna torácica e segurando bastão com os braços estendidos (3 séries de 10 repetições), agachamento isométrico com uso da bola suíça apoiada na região de coluna torácica durante 30 segundos, fortalecimento de isquiostibiais com caneleira de $1 \mathrm{~kg}$ (3 séries de 12 repetições), fortalecimento de quadríceps com caneleira de 1 $\mathrm{kg}$ (3 séries de 12 repetições), fortalecimento isométrico de adutores com o uso de círculo mágico. (2 séries de 1 minuto).

\section{Quarta sessão:}

$\rightarrow$ Aquecimento (8'): Esteira com velocidade média de 2.5)

$\rightarrow$ Exercícios de mobilidade pélvica sentado na bola suíça - (1 minuto cada exercício com intervalo de 30 segundos): Laterização, anteversão, retroversão e circundução.

$\rightarrow$ Exercícios de propriocepção - 3 séries de 1 minuto com intervalo de 20 segundos: Descarga de peso alternando os pés.

$\rightarrow$ Exercícios de dupla tarefa ( 3 rodadas de 1 minuto e 30 segundos cada rodada): Com cones coloridos, sempre dificultando a cada rodada, primeiro só com a mão de 
qualquer lado, em seguida mão direita ou esquerda e pra finalizar, mão e pé simultaneamente de acordo com as respectivas cores solicitadas e os comandos dados para a paciente.

$\rightarrow$ Fortalecimento muscular de membros inferiores com caneleiras de $1 \mathrm{~kg}$. (3 séries de 12 repetições): Fortalecimento de abdutores e adutores, fortalecimento de quadríceps, fortalecimento de isquiostibiais.

$\rightarrow$ Eletroterapia (6'): Ultrassom na região de fossa poplítea no modo pulsado, frequência $1 \mathrm{MHz}$, pulso de $100 \mathrm{~Hz}$, intensidade $1 \mathrm{~W} / \mathrm{cm}^{2}$.

\section{Quinta sessão:}

$\rightarrow$ Aquecimento (6'): Bicicleta ergométrica.

$\rightarrow$ Exercícios de dupla-tarefa (1minuto com intervalo de 30 segundos - 3 repetições): Marcha frontal e lateral, passando entre os cones e passando a bolinha por traz do corpo, subir no step e realizar abdução horizontal de ombro com faixa elástica, paciente no centro de 4 cores (preto, azul, vermelho e verde) e o terapeuta realiza o comando de qual membro e qual cor deve ir.

$\rightarrow$ Exercícios de propriocepção (1minuto com intervalo de 30 segundos - 3 repetições): Paciente em apoio bipodal e unipodal em cima do bozu.

$\rightarrow$ Fortalecimento muscular (1 minuto com intervalo de 30 segundos -3 repetições): Sentada na cadeira com bola entre os joelhos e caneleira de $1 \mathrm{~kg}$, realiza flexão de joelho - unilateral, em pé com bola suíça posicionada entre a região torácica e lombar, realiza flexão de quadril e joelhos - agachamento, deitada com quadril e joelhos flexionados com bola entre os joelhos, realiza adução de quadril, apertando a bola, deitada com quadril e joelhos flexionados com faixa elástica acima dos joelhos, realiza abdução de quadril, deitada com quadril e joelhos flexionados, realiza elevação pélvica - ponte.

$\rightarrow$ Eletroterapia (6'): Ultrassom na fossa poplítea com os parâmetros: pulsada, frequência $1 \mathrm{MZ}$, intensidade $1 \mathrm{w} \mathrm{cm} 3$.

\section{Sexta sessão:}

$\rightarrow$ Aquecimento (8'): Bicicleta ergométrica. 
$\rightarrow$ Exercícios de dupla tarefa ( 1 minuto com intervalo de 30 segundos -3 repetições: Paciente no centro de 4 argolas de cores diferentes (rosa, laranja, verde e amarelo) e o terapeuta realiza o comando de qual membro e qual cor dever ir, passando bolinha por trás do corpo, sentada com 4 cones de cores diferentes (rosa, laranja, verde e amarelo) em frente aos pés, terapeuta realiza comando de qual membro e qual cor deve ir.

$\rightarrow$ Exercícios de propriocepção (1minuto com intervalo de 30 segundos - 3 repetições): Paciente em apoio bipodal e unipodal em cima do bozu, paciente em apoio bipodal e unipodal em cima do disco proprioceptivo.

$\rightarrow$ Fortalecimento muscular (1minuto com intervalo de 30 segundos -3 repetições): Sentada na cadeira com bola entre os joelhos e caneleira de $1 \mathrm{~kg}$, realiza flexão de joelho - unilateral, em pé com bola suíça posicionada entre a região torácica e lombar, realiza flexão de quadril e joelhos - agachamento, deitada com quadril e joelhos flexionados com bola entre os joelhos, realiza adução de quadril, apertando a bola, deitada com quadril e joelhos flexionados com faixa elástica acima dos joelhos, realiza abdução de quadril, deitada com quadril e joelhos flexionados, realiza elevação pélvica - ponte.

$\rightarrow$ Eletroterapia (25'): TENS com eletrodos posicionados acima da patela e acima da fossa poplítea, com intensidade inicial 43 e intensidade final 49.

\section{RESULTADOS}

Quadro1: Resultados pre e pós-intervenção

\begin{tabular}{|l|l|l|}
\hline BERG & 45 inicial & 51 final \\
\hline Alcance funcional & $11,5 \mathrm{~cm}$ inicial & $31 \mathrm{~cm}$ final \\
\hline
\end{tabular}

Fonte: autores,2021

Após o total de 6 sessões de fisioterapia, realizadas ao longo de 4 semanas, foram reavaliados os testes feitos anteriormente. Na escala de equilíbrio de Berg a

paciente obteve pontuação 51 e no teste de alcance funcional teve pontuação de $34 \mathrm{~cm}$. 
Além disso, foi notória a melhora da mobilidade articular e do equilíbrio. Em relação ao quadro álgico, a paciente referiu dor articular no joelho direito em todas as sessões, sendo diminuída após as condutas terapêuticas realizadas no atendimento. No entanto, estas variáveis não foram mensuradas previamente, então permanecem subjetivas.

\section{DISCUSSÃO}

O envelhecimento é um processo dinâmico, progressivo e irreversível, ligado diretamente a fatores biológicos, psíquicos e sociais. Devido ao envelhecimento fisiológico e o aumento da expectativa de vida da população, vem se tornando frequente $o$ aparecimento de doenças crônico-degenerativas e isto gera maior preocupação com a saúde da pessoa idosa, visto que, na maioria das vezes, essas doenças levam a incapacidade física e dependência funcional do idoso (RAMOS BRUM et al., 2013).

No Brasil, estima-se que a população acima de 60 anos seja em torno de 19,6 milhões de habitantes (10,2\% da população total) e projeções futuras indicam que o país será o sexto no mundo em número de idosos, ultrapassando a casa dos 30 milhões de pessoas (FECHINE; TROMPIERI, 2015).

O envelhecimento populacional gera sobrecarga ao sistema de saúde e o idoso portador de doença crônica sem controle e/ou tratamento adequado se torna sujeito a sequelas e incapacidades associadas à doença. Além das doenças degenerativas, surgem alterações fisiológicas, relacionadas ao envelhecimento, como a perda de equilíbrio, diminuição de massa e força muscular, tendência ao desenvolvimento de hipertensão arterial, dentre outras alterações. Esses fatores predispõem o idoso a eventuais quedas e/ou acidentes que podem levá-los a incapacidade física (RAMOS BRUM et al., 2013).

A osteoartrite $(A O)$, ou artrose, é a doença musculoesquelética crônica que mais acomete a população mundial, caracterizada pela perda progressiva da cartilagem articular devido a falta de lubrificação do líquido sinovial nas articulações. Dessa forma, torna-se difícil o deslizamento entre as articulações, causando o atrito e, consequentemente, o desgaste articular. Manifesta-se através de dor, rigidez 


\section{QUFN}

articular matinal, crepitação óssea a atrofia muscular. E uma doença crônica multifatorial que leva a uma incapacidade funcional progressiva e o tratamento deve ser multidisciplinar objetivando a melhora funcional, mecânica e clínica (COIMBRA et al., 2004).

A síndrome da dor trocantérica maior (SDGT), ou bursite trocantérica ou tendinopatia glútea, caracteriza-se por dor no trocânter maior do quadril ou em áreas subjacentes. Apresenta alteração nos tendões dos glúteos médio e mínimo, podendo acarretar em distensão da bolsa trocantérica, incapacitação e limitação funcional grave, interferindo na qualidade de vida e prejudicando as atividades de vida diária e laborativas do indivíduo (CASTRO; SILVA, 2020).

Estudos comprovam que a utilização de exercícios de fortalecimento, marcha e equilíbrio trazem uma melhora da dor, amplitude de movimento, equilíbrio, funcionalidade e da qualidade de vida de pacientes com $O A$ de joelho (HAUENSTEIN et al., 2020). Além disso, recentes estudos apontam que a reabilitação deve incluir fortalecimento muscular de abdutores, extensores e rotadores externos de quadril para melhora da capacidade funcional e alívio da dor, sendo inseridos exercícios com carga concêntrica e excêntrica (CASTRO; SILVA, 2020).

Deve-se focar em exercícios terapêuticos que proporcionem melhora da função muscular e da capacidade aeróbica nos pacientes com $A O$. A diminuição da capacidade aeróbica é consequência da diminuição da função muscular e articular, que, sendo trabalhada, irá repercutir na melhora da capacidade funcional, muscular e aeróbica (COSTA et al).

Além disso, junto com a terapia manual, o uso do ultrassom possui efeito significativo na analgesia e diminuição do uso de medicamentos para o controle da dor (MORGAN. SANTOS, 2011).

\section{CONCLUSÃO}

A fisioterapia vem estabelecendo-se como uma terapia convencional capaz de manejar quadros álgicos em doenças crônicas como estas apresentadas pelo 
EDUCAÇÃO, SAÚDE

ETECNOLOGIA

26 A 28 DE OUTUBRO DE 2021

\section{Q P UFN}

paciente em questão. Após as condutas descritas nesse relato de caso, é possível afirmar que houve melhora no equilíbrio e alcance funcional neste idoso. 
CASTRO, Kamilla Maria Sousa de; SILVA, Erislane Natália de Oliveira. Avaliação e manejo fisioterapêutico na síndrome da dor trocantérica maior: revisão integrativa. BrJP, v. 3, n. 2, p. 170-176, 2020.

COIMBRA, I. B. et al. Osteoartrite (artrose): tratamento. Revista Brasileira de Reumatologia, v. 44, n. 6, p. 450-453, 2004.

COSTA, Carla Yohanna Feitosa et al. IMPACTO DA FISIOTERAPIA NA SINTOMATOLOGIA DE UMA IDOSA COM OSTEOARTROSE: UM RELATO DE CASO.

FECHINE, Basílio Rommel Almeida; TROMPIERI, Nicolino. O processo de envelhecimento: as principais alterações que acontecem com 0 idoso com 0 passar dos anos. InterSciencePlace, v. 1, n. 20, 2015.

HAUENSTEIN, Gabriele et al. PRINCIPAIS TRATAMENTO FISIOTERAPÊUTICOS PARA ARTROSE DE JOELHO. Anais do Seminário Interinstitucional de Ensino, Pesquisa e Extensão, 2020.

JEON, Yong-Jin; KIM, Gyoung-Mo. Comparison of the Berg Balance Scale and Fullerton Advanced Balance Scale to predict falls in community-dwelling adults. Journal of physical therapy science, v. 29, n. 2, p. 232-234, 2017.

JORGE, Matheus Santos Gomes et al. Efeitos da cinesioterapia na osteoartrite de joelho em idosos: revisão sistemática. ConScientiae Saúde, v. 17, n. 1, p. 93$100,2018$.

JORGE, Matheus Santos Gomes et al. Efeitos da cinesioterapia na osteoartrite de joelho em idosos: revisão sistemática. ConScientiae Saúde, v. 17, n. 1, p. 93100,2018

KARUKA, Aline H.; SILVA, José AM; NAVEGA, Marcelo T. Análise da concordância entre instrumentos de avaliação do equilíbrio corporal em idosos. Brazilian Journal of Physical Therapy, v. 15, n. 6, p. 460-466, 2011. LEOPOLDINO, Amanda Aparecida Oliveira et al. Impacto de um programa de fortalecimento muscular dos membros inferiores no equilíbrio e na performance funcional de idosos institucionalizados: um estudo controlado e randomizado. Acta Fisiátrica, v. 27, n. 3, p. 174-181, 2020. 
RAMOS BRUM, Ana Karine et al. Programa para cuidadores de idosos com demência: relato de experiência. Revista Brasileira de Enfermagem, v. 66, n. 4, 2013.

RAYMUNDO, Stela Freitas et al. Comparação de dois tratamentos fisioterapêuticos na redução da dor e aumento da autonomia funcional de idosos com gonartrose. Revista Brasileira de Geriatria e Gerontologia, v. 17, n. 1, p. 129-140, 2014.

ROCHA, Thiago Casali et al. The Effects of Physical Exercise on Pain Management in Patients with Knee Osteoarthritis: A Systematic Review with Metanalysis. Revista brasileira de ortopedia, v. 55, n. 5, p. 509-517, 2020.

SCHWARTSMANN, Carlos Roberto et al. Associação entre bursite trocantérica, osteoartrose e artroplastia total do quadril. Revista Brasileira de Ortopedia, v. 49, n. 3, p. 267-270, 2014.

YAMADA, Eloá Ferreira et al. Exercício terapêutico associado à estimulação elétrica nervosa transcutânea reduz dor e aumenta funcionalidade em indivíduos com osteoartrite de joelho. Ciência \& Saúde, v. 12, n. 2, p. e32041e32041, 2019. 